\title{
Reafirmación de valores éticos, morales y ecológicos en estudiantes de la carrera de medicina
}

\section{Reaffirmation of ethical, moral and ecological values in medicine students}

DOI: $10.46932 / \mathrm{sfjdv} 3 \mathrm{n} 1-096$

Received in: Jan 30st, 2021

Accepted in: Feb 1th, 2022

\author{
María Atocha Valdez Bencomo \\ Dra. en Psicología \\ Universidad Autónoma de Guerrero \\ Facultad de Medicina \\ Av. Solidaridad s/n Acapulco; Guerrero. México \\ E-mail: mariaatocha50@ hotmail.com \\ Laura Sierra López \\ Dra. en Patología \\ Universidad Autónoma de Guerrero \\ Facultad de Medicina \\ Av. Solidaridad s/n Acapulco; Guerrero. México \\ E-mail: Dra_laurasierra@hotmail.com \\ Rosa María Guerra Dávila \\ Dra. en Gastroenterología \\ Universidad Autónoma de Guerrero \\ Facultad de Medicina \\ Av. Solidaridad s/n Acapulco; Guerrero. México \\ E-mail: gudr47@gmail.com
}

\section{RESUMEN}

Actualmente se viven momentos desestabilizadores tanto en México como en el mundo, e igualmente en ellos están gestándose, grandes transformaciones, que van desde el calentamiento global, la economía, las guerras, los problemas en educación, las violencias y la inestabilidad familiar etc. Al respecto de la inestabilidad familiar, cabe mencionar que no es ajena, a lo que le circunda y le impacta desde todos los ámbitos. Tampoco pasan desapercibidos los espacio donde se elucubran y desarrollan las cosas y sucesos más transcendentales en los procesos de aprendizajes y transformación de los jóvenes en la vida. Ineludibles se vuelven estos espacios, para orientar a los jóvenes, así como necesario retomar y reafirmar nuevamente los valores. Sobre todo, en estos tiempos, que se habla de la ausencia de ellos (la desvalorización), en otras palabras, la pérdida de valores en los jóvenes.

Hoy en día, sin duda la mirada esta puesta en estas nuevas generaciones de jóvenes, estos como poseedores de una crítica constructiva en la que se refleja el ente solidario y autónomo, en jóvenes, capaces de contribuir a moldear un mundo sensible, armonioso y justo. Objetivo. Determinar los valores, con los que se identifican los estudiantes de nuevo ingreso a la Facultad de Medicina de la Universidad Autónoma de Guerrero. Material y método. El estudio de corte descriptivo. La población, estudiantes del primer semestre. El Instrumento, cuestionario de valores, aplicado a una población mixta de estudiantes. Los resultados, de 198 estudiantes de primer año, 83 (41.91\%) hombres y $114(57.59 \%)$ mujeres, $1(0.5 \%)$ sin registro. Se obtuvieron 1011 menciones de valores, algunos estudiantes realizaron más de los 5 valores 
solicitados en el registro. De los 83 valores, seleccionaron 74. E incluyeron diez valores más que no estaban presentes en el cuestionario. Conclusión. Se refleja un estado de latencia en los estudiantes, no de ausencia en el manejo de valores.

Palabras clave: Valores, Reafirmación de Valores, Estudiantes, Carrera de Medicina.

\begin{abstract}
Destabilizing moments are currently being experienced both in Mexico and in the world, and great transformations are also taking place in them, ranging from global warming, the economy, wars, problems in education, violence and family instability, etc. Regarding family instability, it is worth mentioning that it is not alien to what surrounds it and impacts it from all areas. Nor do the spaces where the most transcendental things and events in the learning and transformation processes of young people in life are pondered and developed. These spaces become unavoidable, to guide young people, as well as necessary to resume and reaffirm values again. Above all, in these times, there is talk of their absence (devaluation), in other words, the loss of values in young people. Today, without a doubt, the gaze is on these new generations of young people, these as holders of a constructive criticism in which the supportive and autonomous entity is reflected, in young people, capable of contributing to shaping a sensible, harmonious and fair world. . Target. To determine the values with which the new students at the Faculty of Medicine of the Autonomous University of Guerrero identify themselves. Material and method. The descriptive study. The population, first semester students. The Instrument, values questionnaire, applied to a mixed population of students. The results, from 198 first-year students, $83(41.91 \%)$ men and 114 (57.59\%) women, $1(0.5 \%)$ without registration. 1011 mentions of values were obtained, some students made more than the 5 values requested in the registry. Of the 83 values, they selected 74 . And they included ten more values that were not present in the questionnaire. Conclusion. A state of latency is reflected in the students, not of absence in the management of values.
\end{abstract}

Keywords: Values, Reaffirmation of Values, Medicine Career Students.

\title{
I INTRODUCCIÓN
}

El objetivo central de toda educación considerada más allá de las fronteras, que conforman una sociedad abierta y competitiva, esta pensada desde la familia, en donde su proceder sea en términos de intervenir para que los jóvenes sean emocionalmente mas estables, en lo social que su inserción sea de fácil adaptación y en el plano de los valores, sean más éticamente responsable, con capacidad para construirse. La carrera de Medicina posee características especiales por ser una disciplina científica que se centra de forma directa en la atención, prevención e intervención de la salud en las personas. Por ello los valores deben prevalecer como una de las principales premisas en la praxis del estudiante en todo el proceso formativo de la carrera y en el ejercicio de la misma. Por esta razón se piensa pertinente que una institución educativa como lo es Medicina considere a partir de su cultura y en función de la visión y misión, los valores, conductas y actitudes, que orientan la tarea formativa y constructiva de los estudiantes hacia el camino de la excelencia académica a través de la praxis profesional. 
El tema de los valores ha sido una vieja preocupación del ser humano. El cual acepta las cosas, las situaciones dándole valores de si es bueno, justo, bello o útil, y que a la vez calificar como malo, injusto, feo o perjudicial estos planteamientos, el hombre ha tenido constantemente que buscar, respuestas para orientarse en la vida, para encontrar las fuerzas motivacionales que guíen su actividad y conducta. (Fabelo Corso, 2004).

Es por ello que al abordar el tema de los valores nos lleva inmediatamente a la reflexión. Sobre todo, por lo que actualmente se escribe de ellos y porque atañe a los jóvenes. Hoy en día se ha vuelto común escuchar y leer que hay crisis por la pérdida de valores en la juventud. Lo cual es altamente notorio en ellos, ya que se observa y reafirma por sus actitudes conductuales-comportamentales la ausencia de los valores, situación que representa actualmente todo un desafío para la educación, la sociedad, el país y el mundo.

Los valores suplen, en la sociedad, la función que en otras especies desempeñan los instintos biológicos, sobre todo, el de la autoconservación. El hecho de que el género humano haya puesto en peligro su propia supervivencia, es el más claro indicador de la aguda crisis de valores por la que atraviesa. La comprensión de esta crisis, en su sentido más profundo, es un requisito imprescindible para su superación. Y ello presupone indagar en el complejo mundo de los valores humanos(Corso \& Ramón, 2004).

Al respecto de lo citado, no es de extrañarse que preguntas como estas: ¿Qué ha pasado con los valores en los jóvenes? ¿Los jóvenes de hoy no tienen valores?, ¿A los jóvenes no les importa vivirse en valores? ¿Es una pérdida de tiempo con ellos?, minimicen el objetivo de trabajar en la reafirmación de los valores.

Cierto es que estas y otras interrogantes se han hecho ya al respecto, ante las conductas de apatía que muestran y expresan los jóvenes en la vida cotidiana, en las aulas y en la escuela en general, así como la falta de respeto, la irresponsabilidad entre otros, todo ello, ha llevado a los jóvenes a mostrar una falta de compromiso con la sociedad y consigo mismos.

Considerando lo mencionado en el párrafo anterior, cabe decir que son diversos los factores que han influido y siguen influyendo en esta cuestión del manejo de los valores, se mencionan al respecto los factores culturales y psicosociales como los de mayor influencia en un comportamiento negativo y de apatía en los jóvenes estudiantes, conductas donde se ve reflejada desde la anulación en algunos y en otros la total ausencia de los valores.

Ante estos escenarios, en los contextos actuales, por los que atraviesa el país y por ende el estado, se vuelve una necesidad imperiosa retomar la temática de los valores, independientemente de todo lo que se ha dicho y escrito al respecto de esta diada (ausencia de valor - jóvenes) y buscar los mecanismos que 
lleven a sensibilizar a los jóvenes para retomar y fortalecer el uso de los valores, considerándolos como un escudo protector para la salud emocional de la persona.

Hoy en día se considera a una persona saludable cuando ella, está bien en las tres esferas de la salud: salud física, salud emocional, salud social (Ferreria, 2011). Hay otros autores que complementan con la esfera espiritual.

La carrera de medicina de la Universidad Autónoma de Guerrero, debe hacer todo lo que este en sus manos para contribuir al desarrollo de estudiantes con perfiles saludables, ya que son el referente, como profesionales de la salud en cualquier lugar del país, donde se desenvuelvan como médicos.

\section{LOS VALORES Y LA CARRERA DE MEDICINA}

Desde las diferentes disciplinas científico-técnicas que estudian el comportamiento de las personas, las investigaciones que explican las estrategias y/o mecanismos cognitivos que posibilitan las relaciones entre nosotros y los aprendizajes que hacemos, se consensua en definir los primeros años de vida de nuestra especie como determinantes para que la integración de los sujetos en las sociedades sea adecuada a las normas, costumbres y valores humanos ético-morales y ambientales que dichas sociedades postulan como válidos y prioritarios para su propio progreso económico y cultural (Casals \& Trave, 2011).

Para enfatizar lo anterior, cabe decir que, en el ser humano, las normas de conducta se transmiten de padres a hijos, de generación en generación, por medio de la herencia biológica y la herencia cultural; por medio de los gametos que llevan los genes; y por medio del ejemplo. Conforme aumenta el tamaño y complejidad del cerebro, la herencia cultural aumenta en importancia, sin embargo, la herencia biológica controla los aspectos particulares del cerebro en cuanto a tamaño y complejidad; es decir, el límite último de la educación es biológico (Guillen \& Abreu, 2007).

Tan es así, que el ser humano no sólo tiene una facultad cognoscitiva que le sirve para emitir juicios sobre la realidad, sino que es capaz también de emitir juicios de valor sobre las cosas. Al hablar del mundo que le rodea, el hombre se refiere a él no sólo con criterios lógicos o racionales, sino también meta-lógicos, que van más allá de la explicación racional (Tierno, 2011).

La palabra valor, procede del latín y más concretamente del vocablo "valere", que puede traducirse como ser fuerte. Cabe entenderse en uno de los tantos contextos al concepto de valor, para nombrar a las características morales que son inherentes a un sujeto. Los valores son propios de las personas y están por todas partes, es decir, todas nuestras acciones y pensamientos están llenos de valores. Este es un hecho que ha pasado, pasa y pasará siempre (Casals \& Trave, 2011). 
Se concibe a los valores humanos universales, pues son compartidos por todos los seres humanos, cualquiera que sea su religión, su nacionalidad, su cultura, su historia personal, por naturaleza, inducen consideración por las demás personas y su bienestar.

Por lo tanto, los valores en general, siendo estos universales o no, tienen diversas características entre las cuales destacan las siguientes:

Durabilidad: Existen valores con diversos períodos de duración, siendo unos más permanentes que otros. Estos se van reflejando a medida que las personas atraviesan su vida (cambio de un semestre a otro, notorio esto en el $7^{\circ}$ y $8^{\circ}$ que cursan los estudiantes). La práctica de los valores dignifica al ser humano en un sentido amplio; aun cuando, en el tiempo, cada sociedad cambia por el dinamismo propio de ella. Sin embargo, los cambios que se dan dentro del contexto social, determinan el desarrollo de la sociedad y esto a su vez influye de forma indirecta en el desarrollo fisiológico como en el comportamiento actitudinal del individuo.

A decir de los valores son todas las cosas que proveen a las personas a defender y crecer en su dignidad en cuanto a persona, porque indefectiblemente el valor moral conducirá al hombre hacia el bien moral, que como sabemos, es aquello que lo perfecciona, lo completa y mejora (Valores, 2012).

Retomando lo citado en párrafo anterior es que expresamos que la carrera de Medicina, posee características especiales por ser una disciplina científica que se centra de forma directa en la prevención, atención, e intervención de la salud en las personas. Por ello se considera la importancia que revisten los valores en esta disciplina y que estos, deben prevalecer como una de las principales premisas en la praxis del estudiante en todo el proceso formativo de la carrera y en el ejercicio de la misma. Por esta razón se piensa pertinente que una institución educativa como lo es Medicina, considere a partir de su cultura y en función de la visión y misión, los valores, conductas y actitudes, que orientan la tarea formativa de los estudiantes hacia el camino de la excelencia académica a través de la praxis profesional. El tema de los valores no es algo nuevo, ha sido una vieja preocupación del ser humano. El cual acepta las cosas y las situaciones, dándole el valor de si es bueno, si es justo, si es lindo o útil, y que a la vez también tiene que calificar como malo, injusto, feo o perjudicial, estos planteamientos. Es así, que el hombre ha tenido constantemente que buscar, respuestas para orientarse en la vida, para encontrar las fuerzas motivacionales que guíen su actividad y conducta. (Fabelo Corso J. R., 2004).

El tema de los valores, nos obliga a la reflexión. Sobre todo, por lo que actualmente se escribe de ellos y porque atañe a los jóvenes. Hoy en día se ha vuelto común escuchar y leer que hay crisis por la pérdida de valores en la juventud. La ausencia de los valores, es altamente notoria en los jóvenes, lo cual se expresa a partir de sus actitudes conductuales-comportamentales. Esta situación, representa actualmente todo un desafío para la educación en todos los niveles, la sociedad, el país y el mundo. 
Los valores suplen, en la sociedad, la función que en otras especies desempeñan los instintos biológicos, sobre todo, el de la autoconservación. El hecho de que el género humano haya puesto en peligro su propia supervivencia, es el más claro indicador de la aguda crisis de valores por la que atraviesa. La comprensión de esta crisis, en su sentido más profundo, es un requisito imprescindible para su superación. Y ello presupone indagar en el complejo mundo de los valores humanos(Corso \& Ramón, 2004).

Considerando lo mencionado en el párrafo anterior, cabe decir que son diversos los factores que han influido y siguen influyendo en esta cuestión del manejo de los valores, se mencionan al respecto los factores culturales y psicosociales como los de mayor influencia en un comportamiento negativo y de apatía en los jóvenes estudiantes, conductas donde se ve reflejada desde la anulación en algunos y en otros la ausencia de los valores.

Ante estos contextos actuales, por los que atraviesa el país y el mundo, se vuelve una necesidad imperiosa, retomar la temática de los valores, independientemente de todo lo que se ha dicho y escrito al respecto de esta triada (ausencia de valor familia- jóvenes) y buscar los mecanismos que lleven a sensibilizar a los jóvenes para retomar y fortalecer el uso de los valores, considerándolos como un factor resiliente, que permita mantener una homeostasis tanto en las salud emocional como fisiológica de la persona.

Hoy en día se considera a una persona saludable, cuando está bien en las tres esferas de la salud: salud física, salud emocional, salud social(Ferreria, 2011).

La carrera de medicina de la Universidad Autónoma de Guerrero, debe hacer todo lo que este en sus manos, para contribuir al desarrollo de estudiantes con perfiles deseable, pero sobre todo saludables, ya que son el referente, como profesionales de la salud en cualquier lugar del país y del mundo. Una de las formas, para que el estudiante desarrolle su autoconcepto o su sí mismo, es reafirmando los valores que permanecen latentes en él, imprimiéndoles un nuevo significado para su tiempo y haciéndolos visibles en su espacio vital, a través de llevarlos a la praxis tanto en el ámbito educativo, como escenarios de primer impacto y transformación.

Cabe resaltar que se retoman los valores humanos éticos, morales y ecológicos como ejes medulares, en el desarrollo individual y social del estudiante de medicina dentro y fuera de la institución.

En tal sentido se busca que, al implementar la práctica de los valores, se mejoren en el estudiante (visto como persona), las condiciones de salud emocional y por ende, se fortalezca el autoconcepto y autoestima, elementos sustanciales que coadyuvan al desarrollo de una mejor calidad de vida. Por ello se considera necesario y apremiante alentar y motivar a los estudiantes, para asumirse en el desarrollo de los valores que están latentes en ellos, que los hagan conscientes y que los resignifiquen (bloqueados, 
reprimidos o cancelados) y que reconsideren la importancia que estos tiene en la construcción y deconstrucción de su personalidad como individuos y entes sociales en transformación.

A demás de abonar para una cultura de la praxis en valores, en el contexto social del cual forma parte. Todo ello sin duda, permitirá reforzar en unos, y en otros desarrollar, de forma gradual y positiva el autoconcepto, siendo este un elemento importante en la estructura de la personalidad del sujeto, (estudiante) que lleva a mantener un nivel de autoestima alta y el desarrollo de una actitud asertiva, dentro y fuera de la comunidad estudiantil de la Facultad de Medicina y sobre todo en el desarrollo de su quehacer profesional.

\section{METODOLOGIA}

Estudio descriptivo, cualitativo-cuantitativo. La población 198 estudiantes de primer semestre de dos generaciones de la carrera de Medicina de la UAGro. Se les solicitó participar en un ejercicio para identificar cuáles eran sus valores más importantes. El instrumento la caja de pandora (GPS) (C.B, 2018) con 83 valores generales, ordenados alfabéticamente. Criterios de selección: Estudiantes legalmente inscritos, en relación al criterio de exclusión: los que no aparecen en lista y que no cuentan con matrícula.

En cuanto al procedimiento la indicación fue decirles: Piensa en tus valores como si fueran tu sistema de orientación, que te guiará a tu siguiente destino" De la tabla de valores marca las 5 valores que te parezcan más relevantes para tu formación y como persona, y además puedes añadir a la lista cualquier otro valor que sientas que debería estar en la tabla.

Hacer aflorar el valor a través de una autoevaluación es, por tanto, la primera tarea que debe acometerse, pues no hay posibilidad de cambiar nada en el estudiante si primero no se acepta en la conciencia. Él momento de la aplicación, fu durante el curso de inducción que se realiza en la semana previa al inicio de

clase, los datos fueron capturados en el programa de Excel, y se consideró para el análisis, el sexo, frecuencia de las menciones de valores registradas y se seleccionaron los diez valores más frecuentes, en la población total y por sexo.

\section{RESULTADOS}

Se presentan los hallazgos obtenidos a partir de la aplicación del instrumento la Caja de Pandora con los 83 valores.

Participaron 198 estudiantes de primer año, 83 hombres (41.91\%) y $114(57.59 \%)$ mujeres, 1 $(0.5 \%)$ sin registro. 
Se obtuvieron 1011 menciones, ya que algunos estudiantes realizaron más de los 5 registros solicitados.

De los 83 valores generales sugeridos, los estudiantes seleccionaron solamente 74 valores, los nueve restantes que no tuvieron ningún registro, fueron: apertura, artisticidad, credibilidad, diversidad, individualidad, influencia, inventiva, riqueza y veneración.

Por otra parte, los estudiantes incluyeron diez valores más, éstos fueron: Altruismo, amabilidad, caridad, entusiasmo, humanidad, humildad, paciencia, respeto, solidaridad y tolerancia.

Así mismo se identifican también los valores que recibieron una sola mención cada uno: Confiabilidad, Ganar, Independencia, Logro, Civismo, Diversión, Fama, Precisión y Tiempo de paz.

A continuación, se presentan los resultados de los valores que fueron seleccionados por los estudiantes en orden de frecuencia, los cuales se expresan en la siguiente figura1. 


\section{Fig. 1. Valores seleccionados por orden de} frecuencia. $\mathbf{N}=1011$

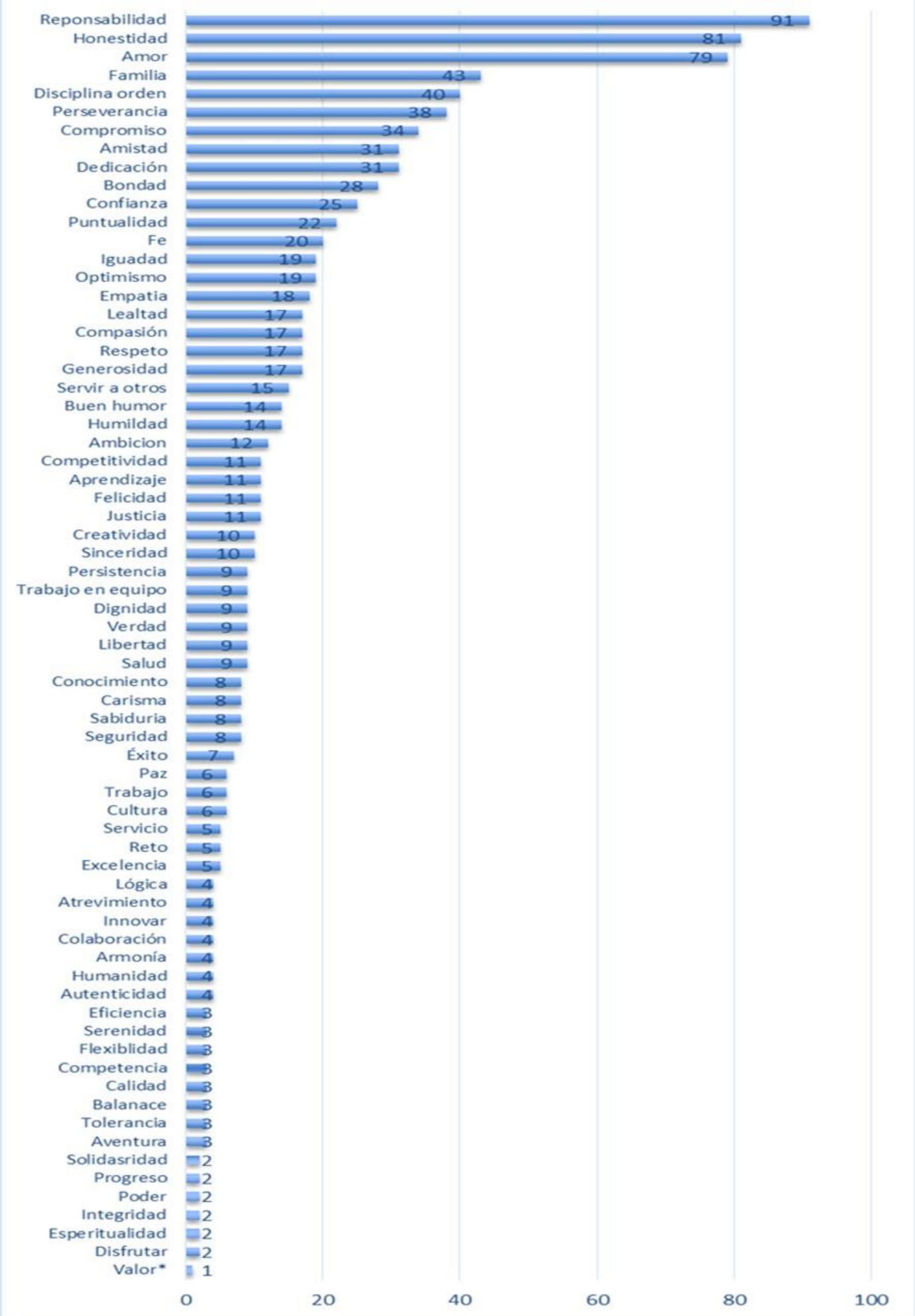

En la población en general, los diez valores con el mayor porcentaje de menciones, alcanzaron el $49.06 \%(\mathrm{n}=496)$ de los 1011 registros. De estos valores, Responsabilidad, Honestidad y Amor, corresponden al $50.60 \%$ de los primeros diez valores y al $24.82 \%$ de todos los registros. 
Los valores seleccionados con mayor número de menciones se expresan en la figura 2.

\section{Fig. 2. Valores seleccionados con mayor número de menciones. $(n=496)$}

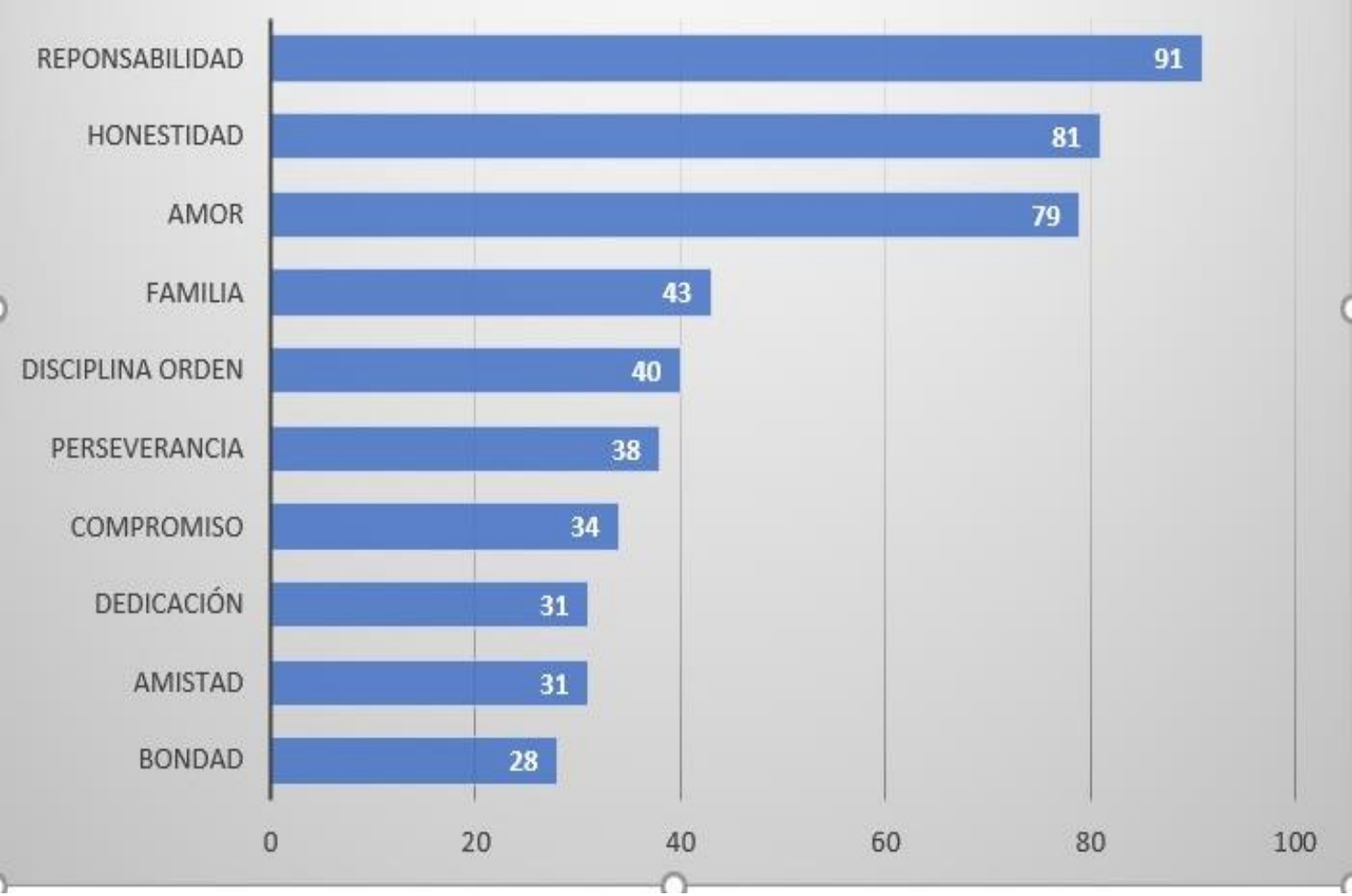

Los siguientes Diez valores seleccionados por los hombres, y de mayor número de menciones se pueden observar en la figura 3.

En cuanto a la distribución por sexo. En los hombres 187 (43.90\%) registros, constituyeron los 10 valores más frecuentes.

En relación a toda la población, se conserva el orden de los primeros cuatro valores, y en el quinto sitio, la disciplina/orden se sustituye por amistad, que en la tabla general aparece en el noveno lugar, ese lugar es sustituido por igualdad y aparece confianza en el décimo sitio. 


\section{Fig. 3. Diez valores seleccionados por los hombres, por mayor número de menciones. $n=187$}

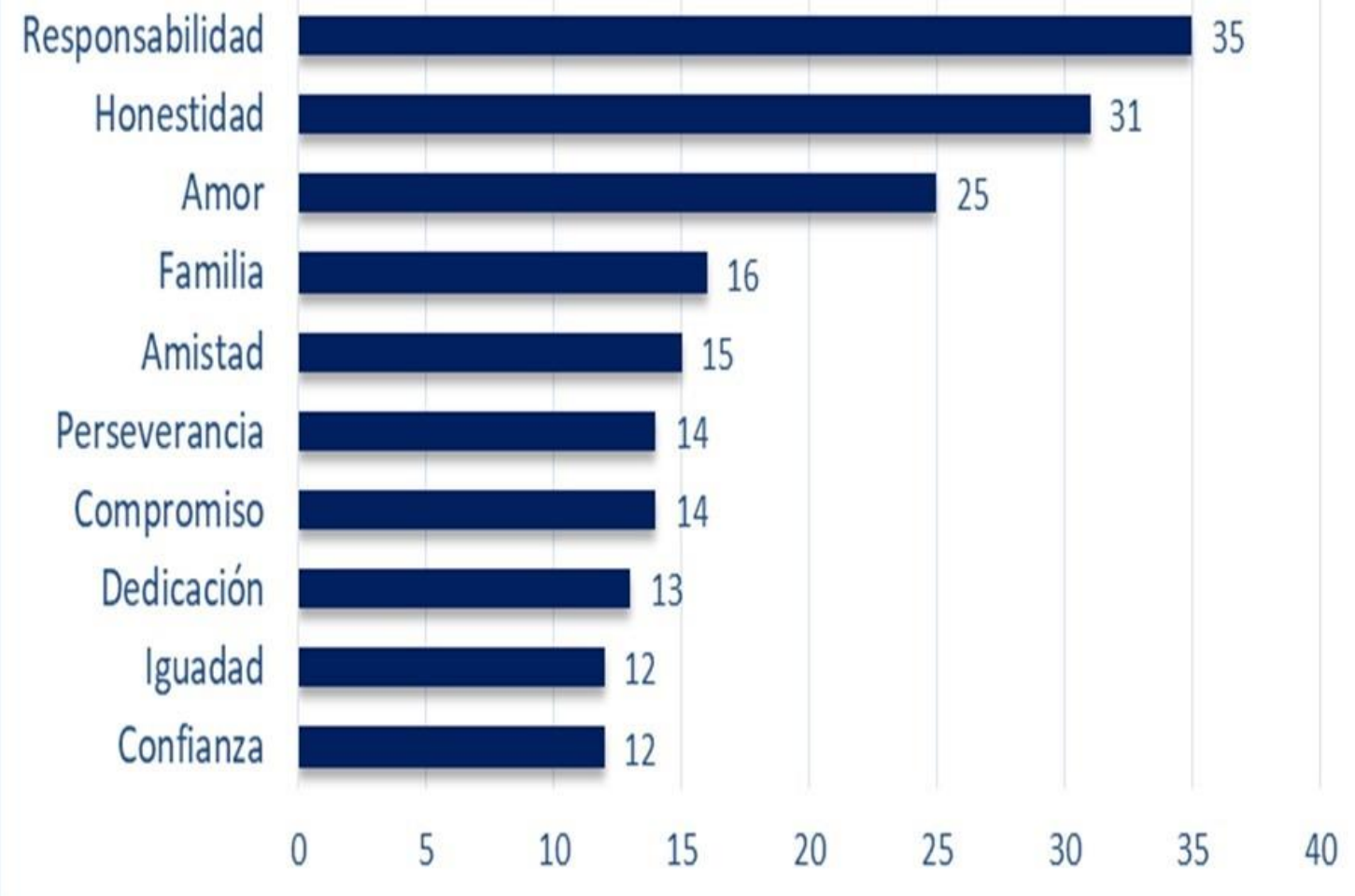

Los siguientes son los Diez valores seleccionados por las mujeres, y los de mayor número de menciones. Como se puede observar en la figura 4.

En las mujeres. El valor del amor, que ocupa el tercer sitio, pasa al segundo, la disciplina/orden y perseverancia, ascienden a 4o y 50 y el valor de la familia se desplaza da 4o a 6o, bondad pasa de 10 a 80. 


\section{Fig. 4. Diez valores seleccionados por las mujeres, por mayor número de menciones. $\mathrm{n}=\mathbf{3 0 8}$}

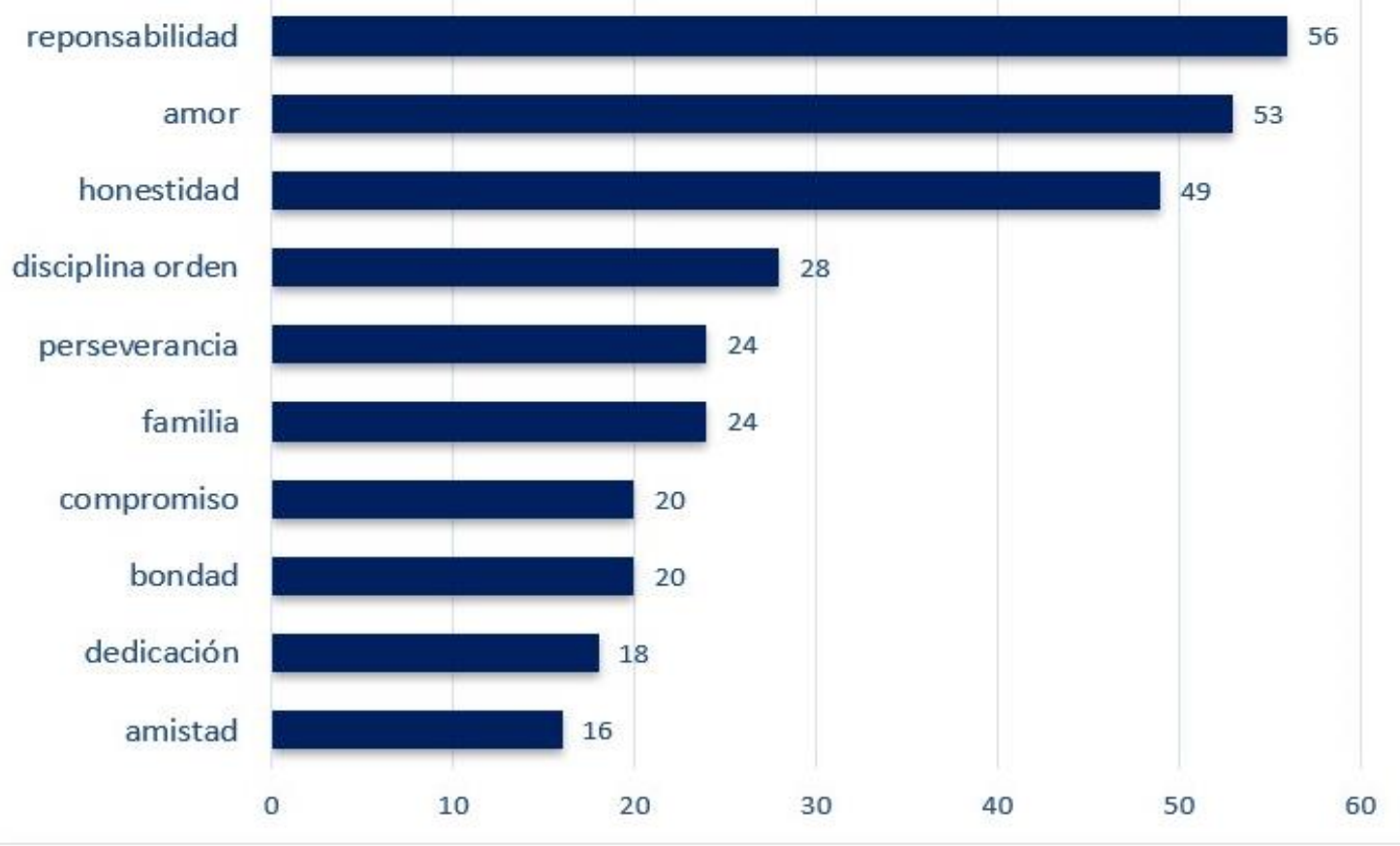

\section{CONCLUSION}

Los estudiantes, no tienen introyectados los valores como elementos sustanciales en su autoconcepto, más los hallazgos, demuestran que estos valores en los jóvenes permaneces latentes por lo tanto es necesario trabajar arduamente en el reforzamiento y motivación en los estudiantes para reafirmarlos. Comparado con nuestro estudio hay resultados coincidentes publicados en México como el de Sánchez y Silva, (2018)' que reportan datos sobre el valor de la honestidad con (65\%), la Familia (63.85), la responsabilidad con (52.9), que se acercan a los valores encontrados en los estudiantes de Medicina de la UAGro.

Así mismo existen otras investigaciones en países como Cuba, que arrojan resultados similares al nuestro, en el trabajo de Seijo Echevarría - J. Finlay y Cols. que reportan el valor de la Honestidad $(89.23 \%)$ y en responsabilidad un (84.72\%). Cabe destacar que los valores de honestidad y responsabilidad en estos trabajos citados y similares en resultados a los encontrados en nuestro estudio, tiene correspondencia con el grupo de valores éticos, y en el grupo de valores morales solo se ubica el valor de la familia.

De los 10 principales valores que arrojo nuestro estudio, los más altos: son la Honestidad, Responsabilidad y Amor. En cuanto a los otros valores como la familia, la perseverancia, el compromiso, 
la dedicación, la amistad, la bondad y la confianza, se observa que los estudiantes tienen gran dificultad para la asimilación e interiorización de ellos.

Por lo que se considera como imperioso trabajar con los estudiantes en la incorporación y reafirmación de los valores en general y en específico de aquellos valores que son necesarios en el desarrollo académico y la formación del perfil en los estudiantes de la carrera de medicina de la UAGro.

Cuadro 10 principales valores en el estudiante de medicina

\begin{tabular}{|l|l|l|l|}
\hline \multicolumn{1}{|c|}{ Valor humano } & \multicolumn{1}{|c|}{ Valor ético } & \multicolumn{1}{c|}{ Valor moral } & V. Ecológico \\
\hline Amor & Responsabilidad & Familia & \\
\hline Bondad & Honestidad & Perseverancia & \\
\hline Amistad & & Compromiso & \\
\hline & & Dedicación & \\
\hline & & Confianza & \\
\hline
\end{tabular}

Fuente: elaboración propia

Como se puede observar en este cuadro, la orientación de los estudiantes de medicina de nuevo ingreso, está dada hacia el manejo de los valores morales a la hora de actuar. Dichos valores tienen que ver con prácticas y costumbres comunes a una sociedad y que tienen que ver con la forma correcta de su actuar. 


\section{REFERENCIAS}

C.B. (2018). Nuestros valores, nuestro GPS interno. Obtenido de https://covabertrand.com/valores-gpsinterno/: https://covabertrand.com/valores-gps-interno/

Casals, E., \& Trave, C. (2011). La educacion en valores. Organizacion de Estados Iberoamericanos.

Corso, F., \& Ramón, J. (2004). Los valores y sus desafios actuales. Cuba: Libros en Red.

Fabelo Corso, J. R. (2004). Los valores y el desafio actual. Habana Cuba: Libros en Red.

Fabelo Corso, J. R. (2004). Los valores y el desafio actual. Habana Cuba: Libros en Red.

Ferreria, R. (22 de Agosto de 2011). Medicina General. Obtenido de IntraMed: http://www.intramed.net/contenidover.asp?contenidoID=72079

Freudenberger, H. (1974). Stsff Burnout. Journal of Social Issues Vol. 30, 159-165.

Guillen, A., \& Abreu, J. L. (2007). Perspectivas de valores con enfsis en valores ecologicos. Daena: Inernational Journal of Good Coonscience. 2 (1) ISSN 1870-557X, 89-97.

Sampieri, H. R., \& Baptista, E. a. (2014). Metodología de la Ivestigación. México: McGrawHill.

Tierno, J. B. (2011). Valores Humanos Vol. I.

Valdez, B. M., Sierra, L. L., \& Et al. (2013). Factores psicosociales e influencia en la desercion y el bajo rendimiento académico. X encuentro Participacion de Mujeres en la Ciencia (pág. 121). Leon, Guanajuato: Centro de Investigaciones en Optica. CIO.

Valores, (C) 2. (martes de marzo de 2012). Recuperado el 7 de Marzo de 2017, de http://www.valoresmorales.net/2012/08/cuales-son-los-valores-morales/ 